\title{
Extensible Markup Language Data Element
}

National Cancer Institute

\section{Source}

National Cancer Institute. Extensible Markup Language Data Element. NCI Thesaurus. Code C142548.

A component of an extensible markup language (XML) document that is used to encapsulate pieces of data and may be delimited by start and end tags or an empty element tag. 